\title{
Design and application of Battery Electric Vehicle (BEV) power information acquisition system based on CAN bus
}

\author{
Wan Nana \\ Automotive Engineering institute, Jiangxi University of Technology, Nanchang 330098, China
}

Keywords: Battery Electric Vehicle; CAN bus; Power information acquisition system; System Desgin

\begin{abstract}
. electric vehicle refers to the vehicle whose electric motor is driven in whole or in part by electric energy as power system. The most common classification includes Battery Electric Vehicle (BEV), Fuel Cell Vehicle (FCV) and Hybrid Electric Vehicle (HEV). The paper designs and applies one electric vehicle battery testing system on account of the characteristic of series battery pack in BEV and shortcomings of common battery test method. The system mainly includes isolated examination of EV battery pack voltage using the way of "bridge capacitor", designing hardware circuit of battery test system, finishing the manufacture and debugging of hardware circuit, solving the systematic sampling, communication and other related issues in the process of debugging, ensuring the stability of the system, designing and applying the master management and control software in battery testing system.
\end{abstract}

\section{Introduction}

Oil and coal is the traditional energy of human; the energy is not only limited, but also pollutes the environment. With the steady growth of economy, the increase of population, the improvement of people's living standards, energy consumption per capita gradually increases and traditional energy crisis is more and more serious. The electric vehicle uses all kinds of energy that can be transformed into electricity; it is imperative to vigorously develop electric vehicle in order to get rid of the dependence on traditional energy and improve energy structure.

Electric vehicle was created in Europe, and French and English are the forerunners for the development. In August 1881, the international electronics exhibition held in Paris, Gustave Trouve exhibited one electric tricycle, which is the first electric vehicle in the history; however, there is no photo for the first one. The photo retained for the oldest electric vehicle is the electric tricycle invented by Englishman Aton and Perry in 1882. Gustave Trouve, Aton and Perry are known as the forerunners of electric vehicle. It should be noted that the first electric vehicle was born in 1873 and invented by Englishman Robert Davidson if electric vehicle with single-use battery can be the one. The first electric vehicle can be used made by Davidson is a truck, which is 48 meters long, 1.8 meters wide, and with single-use battery whose reaction is iron, zinc and amalgam with sulfuric acid. The electric vehicle was spread and developed quickly in the US after the appearance in the Europe. The first battery car was born in Iowa of US with the speed of $23 \mathrm{~km} / \mathrm{h}$ in 1890 .

The effective management for the battery pack is of great significance to ensure the safety of the electric car, keep the battery performance, extend battery life, and increase service efficiency. The basic functions of electric vehicle battery management system are as follows: 
To collect the terminal voltage and temperature of each set of batteries of the electric vehicle battery pack in real time.

To collect the charge-discharge current of the whole battery pack.

The automatic alarm function. The automatic alarm function in the battery management system takes effect when one of the batteries in the battery pack is damaged or the voltage is lower for normal use. In this condition, related information will be displayed for operator to replace the battery, so the reliability of the system can be improved and the battery life extended.

Intelligent charging. When the battery is charged, battery management system can determine the charging status of each battery according to the parameters such as current, voltage, temperature and SOC. According to the charging characteristic and maintenance requirement, the charging process of should be controlled in order to realize the intelligent charging.

The communication function. Communication of battery management system and onboard or off-vehicle equipment is one of its important features. According to the requirements of the application, data exchange can use different communication interfaces, such as analog signals, the PWM signal, CAN bus interface.

Battery management system (BMS) is an important part in electric vehicle. The development of electric vehicle started earlier at overseas; researchers have done lots of practical and theoretical research on onboard battery management, and achieved fruitful results. The United States, France, Germany, Korea and Japan have developed their own battery management system.

$\mathrm{EV}$ is the motor vehicle with battery as the power source. Drivers need to understand the performance of the battery in the time of driving, which mainly includes the battery pack residual capacity, current work status and its life.

The working state of the battery mainly refers to the changes of four parameters, such as terminal voltage, working current, temperature and resistance when the battery is at normal use. The so-called "battery test" usually refers to the detection of the former three parameters.

The detection of battery working state is completed by Battery Management System (BMS). The main tasks of BMS are:

To ensure the safety of battery operation;

To ensure the long service life;

To provide users varied battery information.

\section{Bus technology}

It is difficult for the traditional control system to achieve the information exchange among equipments and information exchange with the outside world; it is an "information island". To meet the requirements of modern automatic control technology, achieve the overall information integration in the enterprise, and implement integrated automation, it's necessary to design the communication system that can run at the industrial environment, be reliable in performance and cheap; to form the factory-floor network, realize the multi-point digital communication among field automation equipments and information exchange among floor field equipments and information exchange between work site and outside world. Field bus comes into being driven by this kind of actual demand.

Field bus is the communication network that is fully distributed, fully digital, intelligent, bidirectional, interconnected, multivariable and multipoint and multi-station, used among field instrument, control system and control room. The definition of field bus made by IEC (International 
Electro-technical Commission) is: a kind of communication technology applied in manufacturing field, and realizes communication among field equipments, and bidirectional, serial, and multipoint, digital communication between field equipment and control devices. This is defined by SC65/WG6 of IOC/TC65 that is in charge of the international standardization work for the communication of measurement and control system data. Field bus is one of the hot spots in the automation field, known as the computer local area in automation field. As the basis of industrial data communication network, field bus connects the productive process field control equipments and higher control management. It is not only a basic network, but also a kind of open and new distributed control system.

Automation system network is the trend of development; the field bus technology is deeply influenced by computer network technology. Nowadays, the development of network technology changes rapidly, and some new network technology with significant impact will further mix into the field bus technology. The promising field bus technologies are: software and hardware technology of intelligent instrument and network equipment development; configuration technology, including network topology, network equipment, network interconnection, etc; technology of network management, including network management software, network data manipulation and transmission.

Field bus technology appeared and was developed in the 1980s, which is closely related with the microcomputer, especially the rapid development of embedded system. Due to various reasons, in the period of more than ten years, several kinds of field bus technology had become mature and been applied gradually, showing unique advantage and strong vitality.

In the early 1980s, BOSCH, a German company made a proposal that CAN (Controller Are a Network) can be used to solve the interior complex hardware signal wiring of the car. Today, almost every new car manufactured in Europe is assembled with at least one CAN network system. CAN is one of the leading field bus protocol, which may lead the world serial bus system. In the year of 1999, nearly 60 million CAN controllers were put into use, and more than 100 million CAN chips were sold in 2000. At present, its application range includes not only the automotive industry but also the areas such as process control, textile machinery, agricultural machinery, robots, numerically-controlled machine tool, medical devices and sensors. CAN bus have been widely used because of its unique design, low cost, high reliability, real-time performance, strong anti-interference and so on.

In the early 1990s, BoschCAN specification (version 2.0) is submitted as an international standard. After several arguments, especially the VAN (Vehicle Area Network) put forward by a few French main car manufactures; the CAN 15011898 standard was released in November, 1993. Meanwhile, the highest Baud rate in the physical layer in CAN protocol is defined as 1Mbps. In addition, the error handling in the CAN data transmission was standardized in 15011519-2 in the year of 1995, and 15011898 Standard was expanded as adding the identifier describing 29-digit CAN. Since all the released CAN norms and standards have some errors and completion, Bosch guarantee (still doing) that all CAN chips should follow BoschCAN reference pattern in order to avoid the incompatibility in use.

Because of the characteristic of CAN bus itself, its application range includes not only the automotive industry but also the areas such as engineering industry, textile machinery, agricultural machinery, robots, numerically-controlled machine tool, medical devices, household appliances and sensors. CAN have the international standard, and are recognized as one of the most promising field bus. 


\section{Battery testing system}

The system includes data collection model and main controller. The data collection model includes collection front-end, CPU (Central Processing Unit), and CAN controller. It is shown in the graph 1

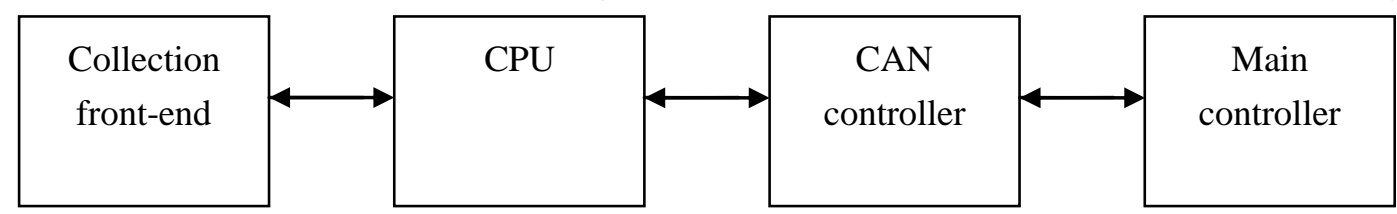

Figure. 1 System overall structure

Generally, EV battery pack works in series system, so the current of single cells and the battery pack is the same; therefore, the detection of one total current value will be fine. The method of testing the terminal voltage of battery pack is simple, only by connecting detection circuit at both ends of battery pack. However, the above method can not be adopted as the terminal voltage of specific single cells can't be determined, although the total working voltage of the battery pack can be determined; moreover, the normal operation and performance of the whole battery pack will be affected due to the problems of only one battery; in addition, the requirement of circuit detection precision is high. The voltage of single cells terminal and its normal operation range are very small. For example, the final voltage of $12 \mathrm{~V}$ lead-acid battery is around $10 \mathrm{~V}$; the voltage change range is from $2 \mathrm{~V}$ to $3 \mathrm{~V}$, and only $10 \%$ of the circuit detection precision can detect the variation of $1 \mathrm{~V}$. When 24 pieces of $12 \mathrm{~V}$ lead-acid battery are connected in series, the rated voltage is $288 \mathrm{~V}$, the final discharging voltage is $240 \mathrm{~V}$, and the normal voltage change range is $48 \mathrm{~V}$. If one battery goes wrong and the terminal voltage decreases to $9 \mathrm{~V}$, the total voltage would be $285 \mathrm{~V}$, with around $1 \%$ change. As you see, the circuit detection precision should be higher than $1 \%$ in order to detect the little voltage change.

It's difficult to find the slow change of single cells in the whole battery pack inspection, such as the aging of single cells itself and accumulation effect brought by consensus problems of single cells. In addition, the terminal voltage of the battery pack has no direct relation with battery capacity (discharge ability).

The performance of single cells and actual capacity of battery pack cannot be detected in whole pack inspection, so the aging cells will not be found.

Generally, the working voltage of electric vehicle motor is as high as $300 \mathrm{~V}$. The dynamic lead-acid battery voltage is $6 \mathrm{~V}$ and $12 \mathrm{~V}$; lithium battery and $\mathrm{Ni}-\mathrm{MH}$ battery have much lower voltage $(4 \mathrm{~V}, 1.2 \mathrm{~V})$, so the number of battery in the battery pack is larger. For example, 24 pieces of battery are needed for $12 \mathrm{~V}$ lead-acid battery providing $288 \mathrm{~V}$ driving voltage. If providing a test module for each battery, the reliability of the system will decline and the cost will increase. Moreover, the capacity of the CAN bus is not enough if the number of battery increases; the nodes number allowed in the CAN bus is 110 . Therefore, the idea of "local concentration" and "overall distribution" was proposed for inspection, namely "concentration/distribution inspection method". According to the method, all batteries are divided into a number of groups, and each group is inspected by "concentration" with inspection module; the whole system is connected by a number of "distribution" inspection modules through CAN bus. In short, the essence of the concentration/distribution inspection system is to detect modularity and localization of units; the data is transmitted by bus. 
The operating temperature of battery is an important parameter to reflect its performance, so the battery status should be detected in time. It is not difficult to collect temperature; the key is how to choose temperature sensor. At present, there are a lot of practical temperature sensors, such as thermocouple, thermal resistance, thermal transistor, integrated temperature sensor, etc. Traditional thermal-sensitive devices are analog signal output, such as the thermocouple, thermal resistance, thermal transistors mentioned above. This kind of sensor has a long history and is widely applied; A/D conversion and signal conditioning are needed when applied in computers. The so-called "conditioning" is to process signal by amplification, filtering, linearization and temperature compensation. The processing is also known as "quadratic transformation", and the circuit completing quadratic transformation is called "processing" circuit.

The system software includes front-end data collection software and main controller software.

The front-end collection module adopts single chip computer P87C591 made by Philips. The single chip computer has the function of DAC (Digital-to-Analogue Conversion), CAN communication, watchdog, etc. The main controller adopts the embedded computer UNO- 2052 of Yanhua Company. The CAN bus of the computer is used to drive the data communication and control of the system. The main tasks of main controller are:

Send the order of collection to data collection module and receive data sent by data collection module when electric vehicles are operating.

Receive the order of collection from charging controller, collect data and send it to charging controller when the battery is charging.

Calculate and process the data collected, and display the data through display screens. The data include battery voltage, circuit current and battery temperature.

Judge the data collected, and alarm when the alarm value reached.

Store the abnormal information of battery so as to analyze the battery performance and remind the battery status.

\section{Conclusion}

Nowadays, the problems of energy shortage and environmental protection are more and more prominent, and the development of electric vehicles is a kind of effective means to solve these problems. The battery performance inspection of electric vehicles concerns the normal, stable and safe operation of electric vehicles. According to the current application status of battery inspection system, the scheme is to construct the battery pack online monitoring system adopting the CAN bus with high reliability, strong anti-interference ability and simple line connection.

\section{Acknowledgements}

This work was financially supported by the key subject building project (vehicle engineering) of Jiangxi University of Technology.

\section{References}

[1] Neubauer J, Wood E. The impact of range anxiety and home, workplace, and public charging infrastructure on simulated battery electric vehicle lifetime utility[J]. Journal of Power Sources, 2014, 257: 12-20. 
[2] Bünte T, Kaspar S, Hohmann S, et al. Inverse model based torque vectoring control for a rear wheel driven battery electric vehicle[C]//IFAC World Congress. 2014.

[3] Donovan M R, Mantzioros T A. A review of the installation and monitoring of Electric Vehicle Charge Points at London Underground car parks[J]. 2014.

[4] Marongiu A, Roscher M, Sauer D U. Influence of the vehicle-to-grid strategy on the aging behavior of lithium battery electric vehicles[J]. Applied Energy, 2015, 137: 899-912.

[5] Zheng Y, Dong Z Y, Xu Y, et al. Electric vehicle battery charging/swap stations in distribution systems: comparison study and optimal planning[J]. Power Systems, IEEE Transactions on, 2014, 29(1): 221-229.

[6] Bi X. Design and Implementation of I/O Modules Based on Dual Redundant Can Bus[C]//2015 International Conference on Mechatronics, Electronic, Industrial and Control Engineering (MEIC-15). Atlantis Press, 2015.

[7] Rong H L, Zhang Y S. Design of Mine Communication Module Based on Power Line Carrier and CAN Bus[C]//Applied Mechanics and Materials. 2015, 733: 775-778.

[8] Tang $\mathrm{Y}$, $\mathrm{Wu} \mathrm{H}$, Liu $\mathrm{H}$. The wireless monitoring system of tower cranes based on MCP2515/CAN bus[C]//2015 International Conference on Intelligent Systems Research and Mechatronics Engineering. Atlantis Press, 2015.

[9] Wu J, Luo F. A CAN Bus Automatic Test System[C]//2015 International Industrial Informatics and Computer Engineering Conference. Atlantis Press, 2015.

[10]Mansor H, Markantonakis K, Mayes K. CAN Bus Risk Analysis Revisit[M]//Information Security Theory and Practice. Securing the Internet of Things. Springer Berlin Heidelberg, 2014: 170-179. 